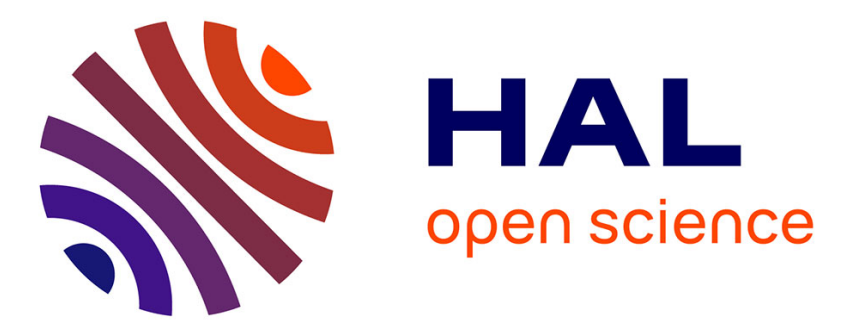

\title{
Nonlinear Robust Coordinated PSS-AVR Control for a Synchronous Generator connected to an Infinite Bus
}

\author{
Cristiano Maria Verrelli, Riccardo Marino, Patrizio Tomei, Gilney Damm
}

\section{To cite this version:}

Cristiano Maria Verrelli, Riccardo Marino, Patrizio Tomei, Gilney Damm. Nonlinear Robust Coordinated PSS-AVR Control for a Synchronous Generator connected to an Infinite Bus. IEEE Transactions on Automatic Control, 2022, 67 (3), pp.1414 - 1422. 10.1109/TAC.2021.3062174 • hal-03482768v2

\section{HAL Id: hal-03482768 \\ https://hal.science/hal-03482768v2}

Submitted on 10 Jun 2022

HAL is a multi-disciplinary open access archive for the deposit and dissemination of scientific research documents, whether they are published or not. The documents may come from teaching and research institutions in France or abroad, or from public or private research centers.
L'archive ouverte pluridisciplinaire HAL, est destinée au dépôt et à la diffusion de documents scientifiques de niveau recherche, publiés ou non, émanant des établissements d'enseignement et de recherche français ou étrangers, des laboratoires publics ou privés. 


\title{
Nonlinear Robust Coordinated PSS-AVR Control for a Synchronous Generator connected to an Infinite Bus
}

\author{
C. M. Verrelli, R. Marino, P. Tomei, G. Damm
}

\begin{abstract}
The transient stabilization and voltage regulation problem for a synchronous generator connected to an infinite bus is addressed, in this technical note, in the presence of all uncertain physical parameters. First, new interpretations for the Desensitized Four Loops Regulator, here referred to as robust coordinated PSS-AVR (Power System Stabilizer \& Automatic Voltage Regulator), are derived in terms of minimum phase properties with respect to a suitably chosen output for the linearized error system. Secondly, on the basis of such an output, a nonlinear generalization of the robust coordinated PSS-AVR is designed with the aim of enlarging the stability region and improving the transients about the unpredictable operating conditions determined by the faults. Its linear action coincides with the one provided by the robust coordinated PSS-AVR: the compelling simplicity of control structure (just one integrator is involved) and robust tuning procedure of the linear design are definitely inherited, with, additionally, no use of the mechanical input power. A numerical analysis along with realistic simulations confirm improved stability when the proposed nonlinear controller is applied.
\end{abstract}

Keywords: Nonlinear control; Synchronous generators; Transient stability; Voltage regulation; Robust coordinated PSS-AVR.

\section{INTRODUCTION}

The problem of power systems stability [1], [20] is still widely studied, according to the latest related theoretical results in [2] and [24], [25], [26], [27]. Transient stability is to be enhanced in the presence of perturbations such as changes in load, outages of powers plants, failures in transformer substation and power lines. Electromechanical oscillations namely, local, inter-machine, and inter-area oscillations - have to be damped, while terminal voltage is to be regulated to its reference value. Linear and local controllers are currently employed. In practice, Linear Automatic Voltage Regulators (AVRs) are used in conjunction with Power System Stabilizers (PSSs) (see [3], [6], [7], [13], [14], [19]). Those controllers are typically numerically designed and tuned on the basis of the classical reduced model of a synchronous generator connected to an infinite bus, whose dynamics are linearized around the (unknown) desired constant operating condition that guarantees terminal voltage regulation. Such a linear model will be referred, throughout this technical note, to

C.M. Verrelli, R. Marino, P. Tomei are with the Electronic Engineering Department of the University of Rome Tor Vergata, Via del Politecnico 1, 00133 Rome, Italy, E-mail (of the corresponding author): verrelli@ing.uniroma2.it. G. Damm is with COSYS-LISIS, Univ Gustave Eiffel, IFSTTAR, F-77447 Marne-la-Vallée, France. as $\mathcal{L} \mathcal{M}$. Even though satisfactory results are achieved by the "Desensitized Four Loops Regulator" DFLR in [3] and [19] (here referred to as robust coordinated PSS-AVR in the whole paper), two open problems remain: $\mathcal{P}_{1}$ : in order to obtain satisfactory performance over a wide range of operating conditions, different behaviours of nonlinear power systems in different operating conditions require different control objectives and consequently different linear control gain scheduling; $\mathcal{P}_{2}$ : the absence of nonlinear feedback terms in non-switching controllers may prevent the enlargement of the machine stability region. Two different strategies are then in order. Switching Linear Controllers: the first strategy consists in using different linearization-based controllers with switching actions depending on the different operating conditions (see [10] and references therein for applications of gain scheduling, fuzzy control and multi-model techniques). Nonlinear NonSwitching Controllers: the second strategy consists in resorting to nonlinear non-switching controllers that incorporate nonlinear feedback terms, simultaneously allowing for the automatic gain scheduling and enlargement of the machine stability region. The structures of such controllers however are rather complex and require additional measurements and parameters. Examples are provided: i) a state feedback solution is proposed in [4] (see also [16], [18]), in the presence of uncertainties in almost all system parameters; ii) output feedback solutions are reported in [22] and [5], in the presence of transmission line impedance and mechanical power uncertainties. In contrast to the robust coordinated PSS-AVR strategy, the strategy adopted in the aforementioned papers (see also [12]) does not consider the terminal voltage as the natural controlled output. Rather, it artificially transforms the power angle into the main controlled output. As a consequence, the (available) terminal voltage regulation error is not directly used in the feedback action, with either the power angle being consequently assumed to be directly measured or the knowledge of critical parameters being required.

This technical note presents two contributions. The first contribution provides new interpretations for the "Desensitized Four Loops Regulator" of [3], by viewing it as a robust stabilizer for the stably invertible system $\mathcal{L M}$ with respect to a suitably chosen output function. The second contribution takes advantage from the previously defined output and presents a new nonlinear controller that generalizes the robust coordinated PSS-AVR - namely, its linear action coincides with the one provided by the robust coordinated PSS-AVR -, with, additionally, no use of the mechanical input power. In fact, such 
a nonlinear generalization just endows the robust coordinated PSS-AVR with: i) a nonlinear term that relies on the power angle (being computed as in [22] or being measured via now available wide-area measurement technologies), allowing for an automatic gain scheduling; ii) nonlinear robustifying terms on the back-stepping-based electrical power tracking error, with the aim - whose effectiveness is illustrated by a numerical analysis - of enlarging the machine stability region. The simplicity of control structure and tuning procedure of the linear design is maintained: just one integrator is involved, while all model parameters are uncertain and are not estimated by the controller. From a theoretical point of view, such a nonlinear contribution simultaneously improves: i) the results in [8], [10], [23], [28], since transient stabilization and voltage regulation tasks are simultaneously accomplished, with no use of robust design strategies that require the existence of suitable stabilizing matrices and with no need of model-knowledgebased feedback linearizing precompensation-actions or switching between transient stabilizers and voltage regulators; ii) the output feedback results in [22] (see also [5] and references therein), since, even when the power angle profile is computed as in [22], only the values for the generator direct axis reactance and the voltage of the infinite bus are here required to be available, whereas several model parameters (including the damping coefficient) are assumed to be known in [22]; iii) the state feedback results in [4] (and all references therein), since several parameter estimates are not required, thus reducing the control complexity. Realistic simulations concerning a benchmark scenario show that the nonlinear controller preserves the stability when a short circuit occurs, whereas instability arises when the standard PSS-AVR is used.

\section{Problem Formulation AND DFLR CONTROL}

In this section we present the generator dynamic model, formulate the problem and introduce the robust coordinated PSS-AVR control (DFLR control).

\section{A. Dynamic model}

The third order dynamic model of a synchronous generator connected to an infinite bus (see [5] for its description and [21], [2] for more general models) is given by:

$$
\begin{aligned}
\dot{\delta} & =\omega \\
\dot{\omega} & =-\frac{D}{H} \omega-\frac{\omega_{s}}{H} P_{e}+\frac{\omega_{s}}{H} P_{m} \\
\dot{P}_{e} & =-\theta_{1} P_{e}+\theta_{2} \omega \sin ^{2}(\delta)+P_{e} \omega \operatorname{cotg}(\delta)+\theta_{3} \sin (\delta) u_{f} \\
V_{t} & =\left[\frac{\theta_{4} P_{e}^{2}}{\sin ^{2}(\delta)}+\theta_{5}+\theta_{6} P_{e} \operatorname{cotg}(\delta)\right]^{\frac{1}{2}},
\end{aligned}
$$

in which: $\delta(\mathrm{rad})$ denotes the generator power angle with respect to the infinite bus rotating at synchronous speed $\omega_{s}$; $\omega=\omega_{g}-\omega_{s}(\mathrm{rad} / \mathrm{s})$ is the difference between the generator angular speed $\omega_{g}$ and the synchronous speed $\omega_{s} ; P_{e}$ (p.u.) denotes the active electrical power delivered by the generator to the infinite bus; $u_{f}$ (p.u.) is the input to the Silicon Controlled Rectifier amplifier of the generator; $P_{m}$ (p.u.) is the mechanical input power, $V_{t}$ (p.u.) is the generator terminal voltage. The reader is referred to [5] for the physical meaning of $D, H$ and the six positive parameters $\theta_{i}>0,1 \leq i \leq 6$. Model (1) turns out to be the well-known single-machineinfinite-bus representation, which, though of low dynamic order, well captures the main (small-signal) characteristics of a synchronous generator in a power network in the presence of parameter variations (see [3], [19]). Now, in order to avoid the physical singularities at $\delta=0$ and $\delta=\pi$, the operation of the system is restricted to an arbitrarily large compact (connected) set $\mathcal{B}$ contained in the open set:

$$
\mathcal{D}=\left\{\left(\delta, \omega, P_{e}\right):\left(\delta, \omega, P_{e}\right) \in(0, \pi) \times \mathbb{R} \times \mathbb{R}^{+}\right\} .
$$

\section{B. Problem formulation}

Definition 1 (transient stabilization \& voltage regulation): Consider the synchronous generator described by the dynamic model (1), with its operation being restricted to $\mathcal{B}$. Assume that all the model parameters are constant and uncertain. The (robust) transient stabilization and voltage regulation problem for the synchronous generator consists in exponentially regulating to zero: $i$ ) the relative speed $\omega$ (transient stabilization); ii) the error between terminal voltage $V_{t}$ and its reference value $V_{t r}=1$ (р.u.) (voltage regulation).

The problem formulation of Definition 1 is well-posed, since the desired operating condition (at which voltage regulation is achieved) exists. It is given by $\delta=\delta_{s}, \omega=0, P_{e}=P_{m}$, where (see [5] for the physical meaning of the parameters):

$$
\delta_{s}=\operatorname{arccotg}\left[-\frac{V_{s}^{2} X_{d}}{X_{s} P_{m}\left(X_{s}+X_{d}\right)}+\sqrt{\frac{V_{s}^{2} V_{t r}^{2}}{X_{s}{ }^{2} P_{m}{ }^{2}}-1}\right]
$$

belongs to the open set $(0, \pi / 2)$ and guarantees terminal voltage regulation, namely:

$$
\left[\frac{\theta_{4} P_{m}^{2}}{\sin ^{2}\left(\delta_{s}\right)}+\theta_{5}+\theta_{6} P_{m} \operatorname{cotg}\left(\delta_{s}\right)\right]^{\frac{1}{2}}=V_{t r} .
$$

It corresponds to a stable equilibrium for system (1) fed by the open loop control $u_{f s}=\theta_{1} P_{m} \theta_{3}^{-1} \sin \left(\delta_{s}\right)^{-1}$. According to [4], there is also another value $\delta_{u} \in[\pi / 2, \pi)$ for $\delta$ (see also [2]) that guarantees terminal voltage regulation. It however corresponds to an unstable equilibrium for system (1) fed by the open loop control $u_{f u}=\theta_{1} P_{m} \theta_{3}^{-1} \sin \left(\delta_{u}\right)^{-1}$.

\section{Robust coordinated PSS-AVR control (DFLR)}

Let us introduce the following regulation errors: $\tilde{\omega}=\omega$, $\tilde{P}_{e}=P_{e}-P_{m}, \tilde{V}_{t}=V_{t}-V_{t r}$. The classical Desensitized Four Loops Regulator (DFLR) (or, equivalently, the robust coordinated PSS-AVR) in [3] [see also (2)-(3) in [19]] can be then written as:

$$
u_{f}(t)=k_{\omega} \tilde{\omega}(t)-k_{p} \tilde{P}_{e}(t)-k_{v} \tilde{V}_{t}(t)-k_{I} \int_{0}^{t} \tilde{V}_{t}(\tau) \mathrm{d} \tau,
$$

in terms of suitable control parameters $k_{\omega}, k_{p}, k_{v}, k_{I}$. The above DFLR is apparently constituted by the combination of: i) the (transient) stabilizing control action

$$
u_{f, \mathrm{ts}}(t)=k_{\omega} \tilde{\omega}(t)-k_{p} \tilde{P}_{e}(t)
$$


that is represented, according to the second equation of (1), by the transfer function (acting on the Laplace transform of the relative speed $\tilde{\omega})$ :

$$
W_{\mathrm{ts}}(s)=k_{\omega}+k_{p} H \omega_{s}^{-1}\left(s+D H^{-1}\right) ;
$$

ii) the voltage regulating control action

$$
u_{f, \mathrm{vr}}(t)=-k_{v} \tilde{V}_{t}(t)-k_{I} \int_{0}^{t} \tilde{V}_{t}(\tau) \mathrm{d} \tau
$$

that is represented by the transfer function (acting on the Laplace transform of the terminal voltage regulation error $\tilde{V}_{t}$ ):

$$
W_{\mathrm{vr}}(s)=-\left(k_{v}+k_{I} / s\right) \text {. }
$$

As shown by [3], such DFLR can be put, by using straightforward approximations that do not significantly deteriorate the performance of the controller, in the standard AVR-PSS structure IEEE ST1A + PSS1A reported in Figures 2 and 4 of [3], with (5) and (7) mimicking the actions of the PSS and AVR, respectively.

\section{NEW INTERPRETATIONS FOR THE DFLR}

Define, besides the already defined regulation errors $(\tilde{\omega}=\omega$, $\left.\tilde{P}_{e}=P_{e}-P_{m}, \tilde{V}_{t}=V_{t}-V_{t r}\right)$, the power angle regulation error: $\tilde{\delta}=\delta-\delta_{s}$. Write the first order approximated expression of $\tilde{V}_{t}$ (around the desired operating condition $\left[\delta_{s}, 0, P_{m}\right]^{\mathrm{T}}$ ) as $\tilde{V}_{t}=-\beta_{0} \tilde{\delta}+\beta_{1} \tilde{P}_{e}$, where $\beta_{0}$ and $\beta_{1}$ turn out to be positive over the generator operating region (see [28]). We preliminarily state Lemma 1, whose results will be used in the reminder of this technical note.

Lemma 1: The linearized dynamics $\mathcal{L} \mathcal{M}$ of the synchronous generator around the desired operating condition $\left[\delta_{s}, 0, P_{m}\right]^{\mathrm{T}}$ is of relative degree one and minimum phase with respect to the output

$$
y=\tilde{V}_{t}-\mu \omega+\nu \tilde{P}_{e},
$$

where $\mu$ and $\nu$ are reals satisfying $\omega_{s} \mu+\left(\beta_{1}+\nu\right) D>0 \&$ $\beta_{1}+\nu>0$.

Proof: Starting from (1), consider the linearized dynamics $\mathcal{L} \mathcal{M}$ in terms of the state variables: $\tilde{\delta}, \tilde{\omega}, \tilde{P}_{e}$ and the output $y$. By mimicking the first steps in [22], perform the change of coordinates: $z_{1}=\tilde{\delta}, z_{2}=\tilde{\omega}, z_{3}=-D H^{-1} \tilde{\omega}-\omega_{s} H^{-1} \tilde{P}_{e}$ and thus obtain the system expressed in the new coordinates. It is of relative degree one and in reachability form: its transfer function (between the output $y$ and the input $\left.u \triangleq \omega_{s} \theta_{3} H^{-1} \sin \left(\delta_{s}\right)\left(u_{f}-u_{f s}\right)\right)$ can be derived to be:

$$
W_{\mathcal{M}}(s)=\frac{\frac{\left(\beta_{1}+\nu\right) H}{\omega_{s}} s^{2}+\left(\mu+\frac{\left(\beta_{1}+\nu\right) D}{\omega_{s}}\right) s+\beta_{0}}{s^{3}+\left(\frac{D}{H}-\alpha_{2}\right) s^{2}+\left(\frac{\omega_{s}}{H} \alpha_{1}-\frac{D}{H} \alpha_{2}\right) s+\frac{\omega_{s}}{H} \alpha_{0}}
$$

where $\alpha_{0}, \alpha_{1}, \alpha_{2}$ are the reals: $\alpha_{0}=\theta_{3} u_{f s} \cos \left(\delta_{s}\right), \alpha_{1}=$ $\theta_{2} \sin ^{2}\left(\delta_{s}\right)+P_{m} \operatorname{cotg}\left(\delta_{s}\right), \alpha_{2}=-\theta_{1}$ depending on the generator operating condition. Owing to the positive nature of the coefficients characterizing the numerator of $W_{\mathcal{M}}(s)$, the minimum phase property is guaranteed.

We now state Theorem 1.

Theorem 1: Consider the linearized dynamics $\mathcal{L} \mathcal{M}$ of the synchronous generator around the operating condition $\left[\delta_{s}, 0, P_{m}\right]^{\mathrm{T}}$. Let $\mu, \nu$ satisfy the constraints of Lemma 1. Then the application of the $(\mu, \nu)-D F L R\left(k_{y}, k_{y I}>0\right)$ :

$$
u_{f, \mu, \nu}(t)=-k_{y} y(t)-k_{y I} \int_{0}^{t} y(\tau) \mathrm{d} \tau
$$

achieves transient stabilization and voltage regulation, provided that $k_{y}$ is sufficiently large.

Proof: Equivalently express the generalized $(\mu, \nu)$-DFLR (9) as:

$$
\begin{aligned}
u_{f, \mu, \nu}(t) & =-k_{y} y(t)+\hat{u}_{f s}(t) \\
\dot{\hat{u}}_{f s}(t) & =-k_{y I} y(t), \quad \hat{u}_{f s}(0)=0 .
\end{aligned}
$$

Consider the transfer function $W_{\mathcal{M}}(s)$. For the sake of compactness: i) denote by $b_{1}, b_{2}, b_{3}$ the positive coefficients of its numerator and let them constitute the components of a column vector $b$, with the zeroes of the polynomial $p(s)=b_{1} s^{2}+b_{2} s+b_{3}$ all belonging to $\mathbb{C}^{-}$; ii) denote by $a_{1}$, $a_{2}, a_{3}$ the coefficients of its monic denominator and let them constitute the components of the column vector $a$. Accordingly take the realization of $W_{\mathcal{M}}(s)$ in observability form:

$$
\begin{aligned}
\dot{x} & =\left[\begin{array}{lll}
0 & 1 & 0 \\
0 & 0 & 1 \\
0 & 0 & 0
\end{array}\right] x+a y+b u \triangleq A_{c} x+a y+b u \\
y & =[1,0,0] x .
\end{aligned}
$$

Recalling that $b_{1}>0$, the linear change of coordinates $\left[\eta^{\mathrm{T}}, y\right] \triangleq\left[\eta_{1}, \eta_{2}, y\right]$ - with $\eta_{i}=x_{i+1}-b_{i+1} b_{1}{ }^{-1} x_{1}, 1 \leq i \leq 2$, $y=x_{1}$ - can be performed and stability arguments similar to those used in [17], once specialized to the case of constant disturbance compensation, can be used to prove the thesis.

The following Corollary demonstrates that the above $(\mu, \nu)$ DFLR (9) is related to a reparameterization of the DFLR (3).

Corollary 1: Consider the linearized dynamics $\mathcal{L} \mathcal{M}$ of the synchronous generator around the operating condition $\left[\delta_{s}, 0, P_{m}\right]^{\mathrm{T}}$. Let $\tilde{P}_{e 0}, \tilde{\omega}_{0}, \tilde{V}_{t 0}$ denote the initial conditions for $\tilde{P}_{e}(t), \tilde{\omega}(t), \tilde{V}_{t}(t)$ at time $t=0$. Define the constant:

$$
\sigma_{0}=k_{y I}\left(\mu+\frac{\nu D}{\omega_{s}}\right)\left(\frac{\beta_{1}}{\beta_{0}} \tilde{P}_{e 0}-\frac{\tilde{V}_{t 0}}{\beta_{0}}\right)+k_{y I} \frac{\nu H}{\omega_{s}} \tilde{\omega}_{0} .
$$

Then, the $(\mu, \nu)$-DFLR (10) with $\hat{u}_{f s}(0)=\sigma_{0}$ becomes the DFLR (3), with $k_{v}, k_{\omega}, k_{p}$ satisfying: $k_{\omega}=k_{y} \mu+$ $k_{y I} \omega_{s}^{-1} H \nu, k_{p}=k_{y} \nu-k_{y I} \beta_{1} \beta_{0}^{-1}\left(\mu+\nu D \omega_{s}^{-1}\right), \quad k_{v}=$ $k_{y}+k_{y I} \beta_{0}^{-1}\left(\mu+\nu D \omega_{s}^{-1}\right), k_{I}=k_{y I}$.

Proof: Use the $\tilde{\delta}$ and $\tilde{\omega}$ dynamics for the computation of the $y$-integral and write $\tilde{\delta}$ as $\beta_{0}^{-1}\left(\beta_{1} \tilde{P}_{e}-\tilde{V}_{t}\right)$.

Once Lemma 1 and Corollary 1 have been established, a direct guideline in the control parameters tuning for (3) can be easily obtained: it involves just one parameter to be set at last, in accordance with the following corollary.

Corollary 2 (limit behaviour): Consider the linearized dynamics $\mathcal{L} \mathcal{M}$ of the synchronous generator around the operating condition $\left[\delta_{s}, 0, P_{m}\right]^{\mathrm{T}}$. Then, the application of the $(\mu, \nu)$-DFLR (9) with $k_{y I} / k_{y}$ being constantly set to $z$, for $k_{y}$ tending to $+\infty$, makes: i) two of the closed loop eigenvalues converge to the two open loop zeros of $W_{\mathcal{M}}(s)$ (not explicitly depending on $X_{d}^{\prime}, T_{d 0}, K_{c}$ ); ii) one of the closed 
loop eigenvalues converge to $-z$; iii) one of the closed loop eigenvalues tend to $-\infty$ (along the real axis).

Proof: Consider $\mathcal{L} \mathcal{M}$, expressed, in accordance with the proof of Lemma 1, in terms of the coordinates $z_{1}, z_{2}, z_{3}$ and rewrite $u_{f, \mu, \nu}$ in terms of such coordinates. Define the new variable

$$
\begin{aligned}
H z_{4}= & -\omega_{s} \alpha_{0} z_{1}-\left(\omega_{s} \alpha_{1}-D \alpha_{2}\right) z_{2}-\left(D-H \alpha_{2}\right) z_{3} \\
& -\omega_{s} \theta_{3} \sin \left(\delta_{s}\right)\left(u_{f}-u_{f s}\right)
\end{aligned}
$$

and compute its dynamics. The proof relies on the root locus analysis and on the structure of the corresponding characteristic polynomial for the error system in $z_{1}, z_{2}, z_{3}, z_{4}$ coordinates, which reads

$$
q_{z}(s)=m_{1}(s)+k_{y} \omega_{s} \sin \left(\delta_{s}\right) \theta_{3} H^{-1} m_{2}(s),
$$

where the roots of the polynomials $m_{1}(s)$ and $m_{2}(s)$ denote the open loop poles and zeroes, respectively (recall $W_{\mathcal{M}}(s)$ and the Laplace transform of $u_{f, \mu, \nu}$ in (9)). Such open loop zeroes, in accordance with the definition of $\beta_{0}, \beta_{1}$ and $\theta_{4}, \theta_{6}$, $\delta_{s}$, do not explicitly depend on $X_{d}^{\prime}, T_{d 0}, K_{c}$.

The proof of such a corollary clarifies that non-zero $\mu$ and $\nu$ in (9) are assigned to guarantee satisfactory transient performance, and in particular, to satisfactorily damp down speed oscillations: if $\mu=\nu=0$, then, for arbitrarily large $k_{y}$ (and $k_{y I} / k_{y}$ being constantly set to $z$ ), two closed loop eigenvalues are attracted by the open loop zeroes of $W_{\mathcal{M}}(s)$, which, for such $\mu=\nu=0$, guarantee a non-satisfactory damping since $D$ is typically very small. On the other hand, Corollary 2 suggests to choose $\mu, \nu, k_{y I} / k_{y}$ to robustly place the open loop zeroes in the left half complex plane $\mathbb{C}^{-}$ (independently of $X_{d}^{\prime}, T_{d 0}, K_{c}$ ), and then to largely increase $k_{y}$ to achieve transient stabilization and voltage regulation ${ }^{1}$. The different parameterization of the DFLR (3) rather allows, to arbitrarily impose, once (3) is applied to the error system in $z_{1}, z_{2}, z_{3}, z_{4}$-coordinates, the four closed loop eigenvalues through the four control parameters $k_{\omega}, k_{p}, k_{v}, k_{I}$ (to be simultaneously chosen depending on the specific operating condition and all the system parameters).

\section{NONLINEAR GENERALIZATION OF THE DFLR}

In this section, we consider the nonlinear model (1) in place of the linearized one $\mathcal{L M}$. We accordingly present the design of a new nonlinear controller that generalizes, to the nonlinear model (1), the design of the robust coordinated PSS-AVR of Sections II-III, while additionally avoiding the use of the mechanical input power. The proposed nonlinear

\footnotetext{
${ }^{1}$ It is interesting to notice that the industrial coordinated PSS-AVR of Figure 5 of [3] can be, in turn, obtained through the application of a slight variant of the approach underlying the proof of Theorem 1 . Such a variant is based on Lemma 4 in [15]. In particular, while the controller in Theorem 1 (described by a proper rational function) first feeds back the output error $y$ to stabilize the (minimum phase) error system and then includes an integral action on $y$ being responsible of generating a converging estimate of the uncertain constant $u_{f s}$, such a different controller relying on Lemma 4 in [15] (described by a strictly proper transfer function) first includes an integrator in its transfer function and then stabilizes the resulting error feedback through a proper rational function. In this respect, notice that Lemma 4 in [15] is correct for $d \leq 4$, whereas, for $d \geq 5$, a typo concerning $g_{0}$ in place of $q_{d}$ appears (which the counterexample of [11] to Lemma 4 of [15] just relies on).
}

generalization of the robust coordinated PSS-AVR is given by:

$$
\begin{aligned}
u_{f} & =\frac{-k_{p}\left(P_{e}-P_{e r}\right)-k_{r} \Phi\left(\delta, P_{e}\right)\left(P_{e}-P_{e r}\right)+\lambda \dot{\hat{\rho}}}{\sin (\delta)} \\
\Phi\left(\delta, P_{e}\right) & =\left[\sin ^{2}(\delta)\right]^{2}+\left[P_{e} \operatorname{cotg}(\delta)\right]^{2} \\
P_{e r} & =k_{p \omega} \omega+\hat{\rho} \\
\dot{\hat{\rho}} & =-\mu_{v} \tilde{V}_{t}+\mu_{\omega} \omega,
\end{aligned}
$$

where: $k_{p}, k_{r}, \lambda, k_{p \omega}, \mu_{v}$ are suitable positive control parameters; $\mu_{\omega}$ is a suitable non-negative control parameter satisfying $\max \left\{D, \mu_{\omega}\right\}>0$.

Before stating the main result of this section (namely, Theorem 2), the following comments are in order.

Comment A. The nonlinear robust coordinated PSS-AVR (15) involves the injection of the power angle to provide an automatic gain scheduling and to handle the severe disturbances and contingencies characterizing the operation of power systems. We can provide two interpretations for the above controller (15): i) the state feedback one, when the power angle is measured via now available wide-area measurement technologies (see [9]); ii) the output feedback one, when the values of $\delta$ and $X_{s}$ are computed through the $\left(\delta, X_{s}\right)$ formulas derived in [5] ( $Q$ is the reactive power):

$$
\begin{aligned}
\delta & =\operatorname{arccotg}\left[-\frac{V_{s}^{2} X_{d}}{X_{s} P_{e}\left(X_{s}+X_{d}\right)} \pm \sqrt{\frac{V_{s}^{2} V_{t}^{2}}{X_{s}^{2} P_{e}^{2}}-1}\right] \\
X_{s} & =\frac{-Q V_{s}^{2} \pm \sqrt{Q^{2} V_{s}^{4}-\left(Q^{2}+P_{e}^{2}\right)\left(V_{s}^{2}-V_{t}^{2}\right) V_{s}^{2}}}{Q^{2}+P_{e}^{2}}
\end{aligned}
$$

that just require the knowledge of the generator direct axis reactance and the voltage of the infinite bus ${ }^{2}$. With this respect, recall that $X_{s}$ may undergo sudden variations when electrical perturbations, such as faults on the transmission line, occur.

Comment $B$. Set $\mu_{\omega}=0$ and $\hat{\rho}(0)=P_{m}$. Then, when higher order robustifying actions are neglected [w.r.t. the regulation and estimation errors $\left.\tilde{\delta}, \tilde{\omega}, \tilde{P}_{e}, P_{m}-\hat{\rho}\right],(15)$ reduces to $\left(\bar{k}_{p}=\right.$ $\left.k_{p}+k_{r} \Phi\left(\delta_{s}, P_{m}\right)\right)$ :

$u_{f}=\frac{1}{\sin (\delta)}\left[-\bar{k}_{p} \tilde{P}_{e}+\bar{k}_{p} k_{p \omega} \omega-\lambda \mu_{v} \tilde{V}_{t}-\bar{k}_{p} \mu_{v} \int_{0}^{t} \tilde{V}_{t}(\tau) \mathrm{d} \tau\right]$

that coincides with the DFLR (3):

$u_{f}=\frac{1}{\sin \left(\delta_{s}\right)}\left[-\bar{k}_{p} \tilde{P}_{e}+\bar{k}_{p} k_{p \omega} \omega-\lambda \mu_{v} \tilde{V}_{t}-\bar{k}_{p} \mu_{v} \int_{0}^{t} \tilde{V}_{t}(\tau) \mathrm{d} \tau\right]$

once $\delta$ appearing at the denominator is replaced by $\delta_{s}$ and incorporated into the control gains. In other words, the nonlinear robust coordinated PSS-AVR (15) exhibits different linearizations around different operating conditions. Those different linear actions can be seen as equivalent actions of local robust coordinated PSS-AVR linear controllers with different control parameters. The controller (15) can be thus viewed as a nonlinear version of a simple combination between a robust coordinated PSS-AVR and an automatic smooth nonlinear gain scheduling procedure. The operating status of the controller

\footnotetext{
${ }^{2}$ Uncertainties about the infinite bus voltage value are usually present in the practice. In the realistic simulations of Section $\mathrm{V}$, just the nominal value of the infinite bus voltage will be used.
} 
is automatically changed when operating conditions vary, so that a smooth transfer between the actions of local robust coordinated PSS-AVR linear controllers is performed.

We are now able to present Theorem 2.

Theorem 2: Consider the nonlinear robust coordinated PSS-AVR (15). Besides $\tilde{\delta}=\delta-\delta_{s}$ and $\tilde{\omega}=\omega$, define the new error coordinates: $e_{p}=P_{e}-P_{e r}-P_{e s s}, \tilde{P}_{m}=P_{m}-P_{e s s}-\hat{\rho}$ in terms of the suitable constant

$$
P_{\text {ess }}=\frac{-\theta_{1} P_{m}}{\theta_{3}\left[k_{p}+k_{r} \Phi\left(\delta_{s}, P_{m}\right)\right]} .
$$

Then transient stabilization and voltage regulation is achieved. In particular, the origin of the error system in the $(\tilde{\delta}, \tilde{\omega},-(D+$ $\left.\left.\omega_{s} k_{p \omega}\right) H^{-1} \tilde{\omega}+\omega_{s} H^{-1} \tilde{P}_{m}, e_{p}\right)$-coordinates - corresponding to the desired operating condition $\left[\delta_{s}, 0, P_{m}\right]^{\mathrm{T}}$-, is exponentially stable, for sufficiently large $k_{p}$ and $k_{r}$ and for suitable $\mu_{\omega}$, $\mu_{v}, k_{p \omega}$ robustly placing in $\mathbb{C}^{-}$the roots of the polynomial (not explicitly depending on $X_{d}^{\prime}, T_{d 0}, K_{c}$ ): $\Pi_{A}(s)=s^{3}+$ $\left(\left(D+\omega_{s} k_{p \omega}\right) H^{-1}+\beta_{1} \mu_{v}\right) s^{2}+\left(\beta_{1} D H^{-1} \mu_{v}+\omega_{s} H^{-1} \mu_{\omega}\right) s+$ $\omega_{s} \beta_{0} H^{-1} \mu_{v}$.

Proof: First compute the $\tilde{\delta}$ - and the $\tilde{\omega}$-dynamics by defining $\lambda_{\omega}=\left(D+\omega_{s} k_{p \omega}\right) H^{-1}$. According to the first part of Section III, write

$$
\tilde{V}_{t}=-\beta_{0} \tilde{\delta}+\beta_{1} \tilde{P}_{e}+h_{V}\left(\tilde{\delta}, \tilde{P}_{e}\right),
$$

where $h_{V}(\cdot, \cdot)$ collects the higher order terms in the regulation errors $\tilde{\delta}$ and $\tilde{P}_{e}$. By writing $\tilde{P}_{e}=e_{p}+k_{p \omega} \tilde{\omega}-\tilde{P}_{m}$ and by recalling the definition of $P_{e r}$, the $\tilde{V}_{t}$-expression, in the new error coordinates, becomes:

$\tilde{V}_{t}=-\beta_{0} \tilde{\delta}+\beta_{1} k_{p \omega} \tilde{\omega}-\beta_{1} \tilde{P}_{m}+\beta_{1} e_{p}+\eta_{V}\left(\tilde{\delta}, \tilde{\omega}, \tilde{P}_{m}, e_{p}\right)$ where $\eta_{V}\left(\tilde{\delta}, \tilde{\omega}, \tilde{P}_{m}, e_{p}\right) \triangleq h_{V}\left(\tilde{\delta}, \tilde{P}_{e}\right)$ collects, by definition, the higher order terms in the new errors $\tilde{\delta}, \tilde{\omega}, \tilde{P}_{m}, e_{p}$. The injection of the output $y$ into the $\tilde{P}_{m}$-dynamics (no knowledge of the mechanical input power is here assumed, so that $\nu=0$ has to be taken in (8)) is thus able to modify the last row of the matrix characterizing the $\left(\tilde{\delta}, \tilde{\omega}, \tilde{P}_{m}\right)$ - error subsystem. Accordingly write the error dynamics in terms of the new error variables: $z_{1}=\tilde{\delta}, z_{2}=\tilde{\omega}, z_{3}=-\lambda_{\omega} \tilde{\omega}+\omega_{s} H^{-1} \tilde{P}_{m}$. The aim is to recover the previously presented arguments concerning the error system in $z$-coordinates, once: i) the value of $D$ has been artificially modified through the $k_{p \omega}$-based prefeedback action (with the aim of obviating the absence of $\nu$ ); ii) $\dot{\hat{\rho}}$ has been endowed with the role of $u_{f}$ and $y$ has been fed back with the suitable negative sign in order to stabilize, with the desired eigenvalues, the resulting third-order error subsystem. In this sense, the polynomial $\Pi_{A}(s)$ does constitute the mirrored counterpart of the characteristic polynomial of the matrix characterizing $\mathcal{L M}$ in $z$-coordinates, once $u_{f}$ is replaced by $\dot{\hat{\rho}}$. After some tedious computations, the perturbed triangular system:

$$
\begin{aligned}
\dot{z}_{1}= & z_{2} \\
\dot{z}_{2}= & z_{3}-\frac{\omega_{s}}{H} e_{p} \\
\dot{z}_{3}= & -\omega_{s} \beta_{0} H^{-1} \mu_{v} z_{1}-\left(\beta_{1} D H^{-1} \mu_{v}+\omega_{s} H^{-1} \mu_{\omega}\right) z_{2} \\
& -\left(\lambda_{\omega}+\beta_{1} \mu_{v}\right) z_{3}+\omega_{s} H^{-1}\left(\lambda_{\omega}+\beta_{1} \mu_{v}\right) e_{p} \\
& +\omega_{s} H^{-1} \mu_{v} \eta_{z}\left(z_{1}, z_{2}, z_{3}, e_{p}\right)
\end{aligned}
$$

is derived, where $\eta_{z}\left(z_{1}, z_{2}, z_{3}, e_{p}\right) \triangleq \eta_{V}\left(\tilde{\delta}, \tilde{\omega}, \tilde{P}_{m}, e_{p}\right)$. In accordance with the companion form structure of the matrix characterizing the above error subsystem, it is possible to choose $\mu_{v}, \mu_{\omega}, k_{p \omega}$ (affecting $\lambda_{\omega}$ ) in order to robustly place all its eigenvalues - namely, the roots of the polynomial $\Pi_{A}(s)$ - in the left half complex plane $\mathbb{C}^{-}$(independently of $X_{d}^{\prime}, T_{d 0}$, $K_{c}$ ). Meanwhile, notice how $\mu_{\omega}$ is to be necessarily positive when $D$ is zero. On the other hand, the $e_{p}$-dynamics can be derived as:

$$
\begin{aligned}
\dot{e}_{p}= & -\theta_{1} P_{e}+\theta_{2} \omega \sin ^{2}(\delta)+P_{e} \omega \operatorname{cotg}(\delta)+\theta_{3} \sin (\delta) u_{f} \\
& -\left[k_{p \omega}\left(z_{3}-\omega_{s} H^{-1} e_{p}\right)+\mu_{v} \beta_{0} z_{1}-\mu_{v} \beta_{1} e_{p}\right. \\
& +\mu_{v} \beta_{1} H \omega_{s}^{-1} z_{3}+\mu_{v} \beta_{1} H \omega_{s}^{-1} \lambda_{\omega} z_{2}-\mu_{v} \beta_{1} k_{p \omega} z_{2} \\
& \left.-\mu_{v} \eta_{z}\left(z_{1}, z_{2}, z_{3}, e_{p}\right)+\mu_{\omega} z_{2}\right]
\end{aligned}
$$

with $P_{e}$ again satisfying $P_{e}=e_{p}-\tilde{P}_{m}+k_{p \omega} \tilde{\omega}+P_{m}$. Finally get:

$$
\begin{aligned}
\dot{e}_{p}= & -\theta_{1} e_{p}-\theta_{1} P_{m}+\theta_{1} H \omega_{s}^{-1}\left(z_{3}+\lambda_{\omega} z_{2}\right)-\theta_{1} k_{p \omega} z_{2} \\
& +\theta_{2} z_{2} \sin ^{2}(\delta)+P_{e} z_{2} \operatorname{cotg}(\delta)+\theta_{3} \sin (\delta) u_{f} \\
& -\mu_{v} \beta_{0} z_{1}-\mu_{v} \beta_{1} D \omega_{s}^{-1} z_{2}-\mu_{\omega} z_{2} \\
& -\left(k_{p \omega}+\mu_{v} \beta_{1} H \omega_{s}^{-1}\right) z_{3}+\left(k_{p \omega} \omega_{s} H^{-1}+\mu_{v} \beta_{1}\right) e_{p} \\
& +\mu_{v} \eta_{z}\left(z_{1}, z_{2}, z_{3}, e_{p}\right)
\end{aligned}
$$

whereas, from (15), write

$u_{f} \sin (\delta)=-k_{p} e_{p}-k_{r} \Phi\left(\delta, P_{e}\right) e_{p}+\lambda \dot{\hat{\rho}}+\frac{\theta_{1} P_{m}}{\theta_{3}}-k_{r} P_{e s s} \Lambda_{p}$,

with $\Lambda_{p}=\left(\sin ^{2}(\delta)\right)^{2}-\left(\sin ^{2}\left(\delta_{s}\right)\right)^{2}+\left(P_{e} \operatorname{cotg}(\delta)\right)^{2}-$ $\left(P_{m} \operatorname{cotg}\left(\delta_{s}\right)\right)^{2}$. On the basis of the $\Lambda_{p}$-expression and the $P_{\text {ess }}$-definition, write

$$
-k_{r} P_{e s s} \Lambda_{p}=\frac{k_{r} \theta_{1} P_{m}}{\theta_{3}\left[k_{p}+k_{r} \Phi\left(\delta_{s}, P_{m}\right)\right]} \Lambda_{p} \triangleq \alpha_{p} \Lambda_{p},
$$

with $\alpha_{p}$ belonging to the open connected set $\mathcal{C}_{p}=$ $\left(0, \theta_{1} P_{m} \theta_{3}^{-1} \Phi\left(\delta_{s}, P_{m}\right)^{-1}\right)$. Now, in the RHS of (18), let: $A_{z}$ be the matrix multiplying the vector $z=\left[z_{1}, z_{2}, z_{3}\right]^{\mathrm{T}} ; B_{p}$ be the column vector multiplying $e_{p} ; B_{\eta}$ be the column vector multiplying $\eta_{z}\left(z_{1}, z_{2}, z_{3}, e_{p}\right)$. Set the control parameters to satisfy the related assumption of Theorem 2 (concerning the roots of $\Pi_{A}(s)$ ) and let $P_{z}$ be the symmetric positive definite matrix satisfying the Lyapunov equation $P_{z} A_{z}+A_{z}^{\mathrm{T}} P_{z}=-\mathbb{I}$ in terms of the identity matrix $\mathbb{I} \in \mathcal{M}(3, \mathbb{R})$. Furthermore, consider the composite Lyapunov function

$$
\mathcal{W}=z^{\mathrm{T}} P_{z} z+\frac{1}{2} e_{p}^{2}
$$

that satisfies, along the trajectories of the closed loop system, the equality:

$$
\begin{aligned}
\dot{\mathcal{W}}= & -\|z\|^{2}+2 z^{\mathrm{T}} P_{z} B_{p} e_{p}+2 z^{\mathrm{T}} P_{z} B_{\eta} \eta_{z}\left(z_{1}, z_{2}, z_{3}, e_{p}\right) \\
& -\left[\theta_{1}+k_{p} \theta_{3}-k_{p \omega} \omega_{s} H^{-1}-\mu_{v} \beta_{1}\right] e_{p}^{2} \\
& +\alpha_{p} \Lambda_{p} \theta_{3} e_{p}+\lambda \dot{\hat{\rho}} \theta_{3} e_{p}-k_{r} \theta_{3} \Phi\left(\delta, P_{e}\right) e_{p}^{2} \\
& +\theta_{2} \sin ^{2}(\delta) z_{2} e_{p}+P_{e} \operatorname{cotg}(\delta) z_{2} e_{p} \\
& +\mu_{v} \eta_{z}\left(z_{1}, z_{2}, z_{3}, e_{p}\right) e_{p}-\mu_{v} \beta_{0} z_{1} e_{p} \\
& +\left[\theta_{1} D \omega_{s}^{-1}-\mu_{\omega}-\mu_{v} \beta_{1} D \omega_{s}^{-1}\right] z_{2} e_{p} \\
& +\left[\theta_{1} H \omega_{s}^{-1}-k_{p \omega}-\mu_{v} \beta_{1} H \omega_{s}^{-1}\right] z_{3} e_{p}
\end{aligned}
$$


Recall that $a^{2}-b^{2}=(a-b)(a+b)$ for $a, b \in \mathbb{R}$; then write $P_{e} \operatorname{cotg}(\delta)-P_{m} \operatorname{cotg}\left(\delta_{s}\right)=P_{e} \operatorname{cotg}(\delta)-P_{m} \operatorname{cotg}(\delta)+$ $P_{m} \operatorname{cotg}(\delta)-P_{m} \operatorname{cotg}\left(\delta_{s}\right)$, as well as $\tilde{P}_{e}=e_{p}+k_{p \omega} z_{2}-$ $H \omega_{s}^{-1}\left(z_{3}+\lambda_{\omega} z_{2}\right)$. Then obtain $\left|\Lambda_{p}\right| \leq \sum_{i=1}^{3} \gamma_{p i}\left|z_{i}\right|+\gamma_{4}\left|e_{p}\right|$ over $\mathcal{B} \subset \mathcal{D}$, with $\gamma_{i}(i=1,2,3,4)$ being positive reals depending on the diameter of $\mathcal{B}$ but not increasing while $k_{p}$ and $k_{r}$ increase. On the other hand, write

$$
\begin{aligned}
\lambda \dot{\hat{\rho}}= & -\lambda \mu_{v}\left[-\beta_{0} z_{1}+\beta_{1} k_{p \omega} z_{2}-\beta_{1} H \omega_{s}^{-1}\left(z_{3}+\lambda_{\omega} z_{2}\right)\right. \\
& \left.+\beta_{1} e_{p}+\eta_{z}\left(z_{1}, z_{2}, z_{3}, e_{p}\right)\right]+\lambda \mu_{\omega} z_{2} .
\end{aligned}
$$

Equation (23) thus leads to the inequality ${ }^{3}$

$$
\begin{aligned}
\dot{\mathcal{W}} \leq & -\|z\|^{2}+2\|z\||| P_{z}\left\||| B_{p}\right\|\left|e_{p}\right| \\
& +2\|z\|\left\|P_{z}\right\||| B_{\eta} \|\left|\eta_{z}\left(z_{1}, z_{2}, z_{3}, e_{p}\right)\right| \\
& -\left[\theta_{1}+k_{p} \theta_{3}-k_{p \omega} \omega_{s} H^{-1}-\mu_{v} \beta_{1}+\lambda \mu_{v} \beta_{1} \theta_{3}\right] e_{p}^{2} \\
& +\alpha_{p}\left|\Lambda_{p}\right| \theta_{3}\left|e_{p}\right|-k_{r} \theta_{3} \Phi\left(\delta, P_{e}\right) e_{p}^{2} \\
& +\theta_{2} \sin ^{2}(\delta)\left|z_{2}\right|\left|e_{p}\right|+\left|P_{e} \operatorname{cotg}(\delta)\right|\left|z_{2}\right|\left|e_{p}\right| \\
& +\mu_{v}\left|\eta_{z}\left(z_{1}, z_{2}, z_{3}, e_{p}\right)\right|\left|e_{p}\right|+\mu_{v} \beta_{0}\left|z_{1}\right|\left|e_{p}\right| \\
& +\mu_{v} \beta_{0} \lambda \theta_{3}\left|z_{1}\right|\left|e_{p}\right|+\mid \theta_{1} D \omega_{s}^{-1}-\mu_{\omega}-\mu_{v} \beta_{1} D \omega_{s}^{-1} \\
& +\mu_{v} \beta_{1} D \lambda \theta_{3} \omega_{s}^{-1}+\lambda \theta_{3} \mu_{\omega}|| z_{2}|| e_{p} \mid \\
& +\mid \theta_{1} H \omega_{s}^{-1}-k_{p \omega}-\mu_{v} \beta_{1} H \omega_{s}^{-1} \\
& +\mu_{v} \beta_{1} H \omega_{s}^{-1} \lambda \theta_{3}|| z_{3}|| e_{p} \mid \\
& +\mu_{v} \theta_{3} \lambda\left|\eta_{z}\left(z_{1}, z_{2}, z_{3}, e_{p}\right)\right|\left|e_{p}\right| .
\end{aligned}
$$

By finally completing the squares and by setting sufficiently large $k_{p}$ and $k_{r}$ (under given $\mu_{\omega}, \mu_{v}, k_{p \omega}$ ), we obtain, in terms of suitable positive reals $g_{p}$ and $g_{p b}$, the last compact inequality:

$$
\begin{aligned}
\dot{\mathcal{W}} \leq & -\|z\|^{2} / 4-g_{p} e_{p}^{2}+g_{p b}\left|\eta_{z}\left(z_{1}, z_{2}, z_{3}, e_{p}\right)\right|\left(\left|e_{p}\right|\right. \\
& +\|z\|),
\end{aligned}
$$

where $\eta_{z}\left(z_{1}, z_{2}, z_{3}, e_{p}\right)=h_{V}\left(\tilde{\delta}, \tilde{P}_{e}\right)$ just collects the higher order terms of $\tilde{V}_{t}$ in (17). Such a $\tilde{V}_{t}$ is injected into the error dynamics by the control (15), through the gain $\mu_{v}$. Exponential convergence of the $\left(z, e_{p}\right)$-errors is thus achieved for sufficiently small initial $\left(z, e_{p}\right)$-errors: $z_{1}(0)=\tilde{\delta}(0)$, $z_{2}(0)=\tilde{\omega}(0), z_{3}(0)=-\lambda_{\omega} \tilde{\omega}(0)+\omega_{s} H^{-1}\left(P_{m}-\hat{\rho}(0)-P_{\text {ess }}\right)$, $e_{p}(0)=P_{e}(0)-P_{m}-k_{p \omega} \tilde{\omega}(0)+\left(P_{m}-\hat{\rho}(0)-P_{e s s}\right)$, that make, for any $t$ and in accordance with (24): i) $\dot{\mathcal{W}}$ be negative definite; ii) generator states and references belong to $\mathcal{B}$. With this respect, recall that: i) $\gamma_{i}(i=1,2,3,4)$ are positive reals that do not increase while $k_{p}$ and $k_{r}$ increase; ii) $P_{e s s}$ turns out to decrease as $k_{p}$ and $k_{r}$ increase. On the other hand, achieving exponential convergence of the $\left(z, e_{p}\right)$-errors to zero guarantees exponential convergence of $\tilde{\delta}(t)=\delta(t)-\delta_{s}$, $\tilde{\omega}(t)=\omega(t)$ to zero, along with exponential convergence to zero of $\tilde{P}_{e}(t)$ and $P_{m}-\hat{\rho}(t)-P_{\text {ess }}$.

Comment $C$. The design of (15) and the corresponding stability analysis have taken advantage from the $y$-based arguments of Section III, as the $\tilde{P}_{m}$-dynamics has shown. On

${ }^{3}$ Notice that, when $\lambda \theta_{3}=1$, several coefficients disappear from the nondefinite sign terms. This explains the feedforward role of the $\lambda$-term in (15). the other hand, a back-stepping action that is reminiscent of the typical two-time-scale arguments has been adopted ${ }^{4}$. It is confined to the natural definition of the electrical power reference, so that all the uncertainties in the corresponding electrical power tracking error dynamics are compensated by the integrator placed into the upper mechanical subsystem. Nonlinear robustifying actions can be just included at last, through the use of a composite Lyapunov function. In contrast to the fully linearized analyses which Theorem 1 and Corollary 2 rely on, genuinely nonlinear stability tools (applied to the nonlinear model (1) with just local validity) allow us to neglect, in the Lyapunov analysis of Theorem 2, just the nonlinearities concerning the terminal voltage equation, whereas the well-known nonlinearities affecting the electrical power dynamics are explicitly faced by the nonlinear features of the controller (aiming at enlarging the stability region) and by one last control parameter to be set at last.

Comment D. According to Comment $B$, set $\mu_{\omega}=0$, $\hat{\rho}(0)=P_{m}$, replace $\delta$ appearing at the denominator by $\delta_{s}$ and neglect higher order robustifying actions in (15). Then, (15) coincides with DFLR (3) [under equivalent gains], which admits an attraction domain $\mathcal{R}_{1}$ (whose estimate may be provided by linearization arguments), whereas a possibly different attraction domain $\mathcal{R}_{\mathrm{nl}}$ exists (whose estimate is given by the proof of Theorem 2) for the corresponding nonlinear version (15). The effectiveness of (15) in including points in the state space of (1) - that do not belong to $\mathcal{R}_{1}$ - into its attraction domain $\mathcal{R}_{\mathrm{nl}}$ can be inspected through a numerical analysis. Consider the synchronous generator in [22] (no input saturations are considered), whose physical parameter values are: $\omega_{s}=314.159 \mathrm{rad} / \mathrm{s}, D=5$ p.u., $H=8 \mathrm{~s}, T_{d 0}=6.9 \mathrm{~s}$, $K_{c}=1, X_{d}=1.863$ p.u., $X_{d}^{\prime}=0.257$ p.u., $X_{T}=0.127$ p.u., $X_{L}=0.4853$ p.u.. The nominal operating condition $\delta_{s}=1.256 \mathrm{rad}, P_{m}=0.9$ p.u., $\omega=0 \mathrm{rad} / \mathrm{s}$ guarantees $V_{t}=V_{t r}=1$ p.u., with $V_{s}=1$ p.u.. The common control parameters for both the controllers (15) and (3) [under equivalent gains] are (in SI units): $k_{p \omega}=1.5, \lambda=3, k_{p}=1.5, k_{r}=9$, $\mu_{v}=18\left(\mu_{\omega}=0\right)$, while the controller initial conditions are compatible with the operating point (with the feedforward contribution $u_{f s}$ being included in the controllers). Figure 1 concerning the numerical integration of the equations involved in the considered scenario - shows that there exists a set of initial conditions, namely $\delta(0)=0.3 \mathrm{rad}, \omega(0)=-3 \mathrm{rad} / \mathrm{s}$, $P_{e}(0)=2.2$ p.u., that belongs to the stability region $\mathcal{R}_{\mathrm{nl}}$ guaranteed by (15) but not on $\mathcal{R}_{\mathrm{l}}$.

Comment E. The nonlinear robust coordinated PSS-AVR (15) can be seen as the evolution of the nonlinear decentralized robust adaptive controller in [21], which was exclusively designed for transient stabilization, under a rather weak set of assumptions on the remote network dynamics. The inclusion in (15) of the terminal voltage regulation error to drive the mechanical input power estimate (acting as the integral term of (3)) allows (15) to achieve voltage regulation even at permanently perturbed operating points (see the subsequent Figure 2 concerning a $70 \%$-decrease of $P_{m}$ and a double

\footnotetext{
${ }^{4}$ The use of the proposed technique can be extended to similar scenarios concerning nonlinear systems with extended matching uncertainties.
} 

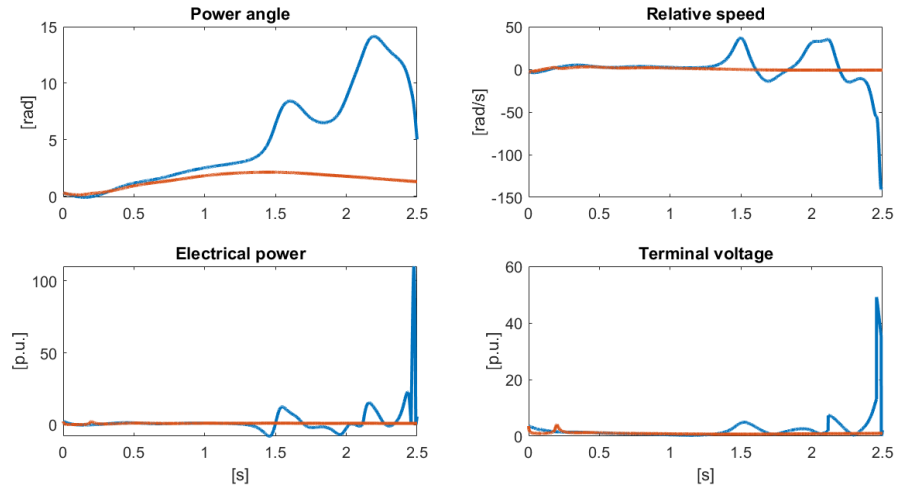

Fig. 1. Power angle $\delta$, relative speed $\omega$, electrical power $P_{e}$, terminal voltage $V_{t}$ (blue: DFLR; red: proposed nonlinear controller).

$33 \%$-increase of the nominal $X_{L}$-value for the synchonous generator already used in Comment D., under the action of the nonlinear controller (15) and the equivalently tuned DFLR (5) with (in SI units): $k_{p \omega}=1.5, \lambda=3, k_{p}=1.5, k_{r}=9$, $\mu_{v}=18\left(\mu_{\omega}=0\right)$, and with the controllers initial conditions being compatible with the operating point. Here the DFLR performance slightly degrades with respect to (15).
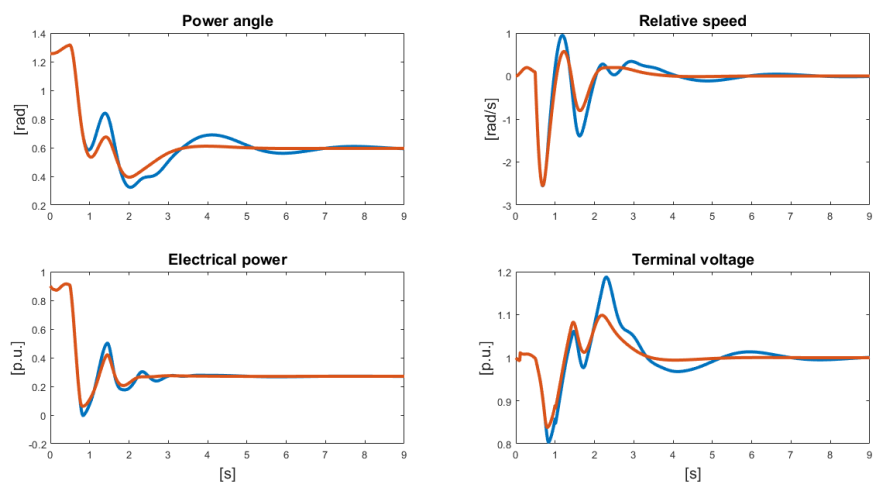

Fig. 2. Power angle $\delta$, relative speed $\omega$, electrical power $P_{e}$, terminal voltage $V_{t}$ (blue: DFLR (3); red: nonlinear controller (15)).

\section{Simulation Results in Simscape Electrical}

This section illustrates the previous theoretical derivations concerning the output feedback version of the control algorithm (15), in comparison to the standard PSS-AVR structure (see Section II.C). The quantities $X_{s}$ and $\delta$ required by (15) have been computed via the $\left(\delta, X_{s}\right)$-formulas of [5], here using $X_{d}$ and just the nominal value of the infinite bus voltage $V_{s}$. The simulations have been carried out with reference to the Matlab/Simulink Simscape Electrical benchmark (including detailed electrical models and several additional elements), in which the duration of the short circuit - placed just after the transformer connecting the test machine to the transmission line - has been set to $90 \mathrm{~ms}$ and no SVC has been considered. In particular, simulations concern the Single Machine Infinite Bus real case at https://fr.mathworks.com/help/physmod/sps/examples/svc-andpss-phasor-model.html, represented by a 1000 MW machine, connected to a $5000 \mathrm{MW}$ power plant through a $700 \mathrm{~km}$ line. The physical saturation for the generators exciters, which is an important limitation for the control performance, is the same for both the controllers. The parameters for the standard PSS-AVR are set as: gain $=2$, wash-out time constant 0.7 , lead-lag time constants $T_{\text {num }}=60 \mathrm{e}^{-3}, T_{\text {den }}=0.5$. The parameters of the output feedback control (15) are chosen as: $k_{p \omega}=70, \lambda=90, k_{p}=10, k_{r}=10, \mu_{v}=10, \mu_{\omega}=10$. Figure 3 shows how the standard PSS-AVR is not able to keep the system stability: $\delta$ goes outbounds. On the other hand, when (15) is used in the test machine - with the infinite bus remaining with the standard PSS-AVR -, systems stability is assured even in the presence of $5 \%$ noise $^{5}$ on the active and reactive electrical powers $P_{e}$ and $Q$, as shown in Figure 4: all states are kept inside their operation bounds. According to Figure 5, the standard PSS-AVR input saturates while being not able to bring the generator back to synchronism to the main grid, whereas the (15)- control input saturates as well but it is then able to bring the generator back to its equilibrium point.
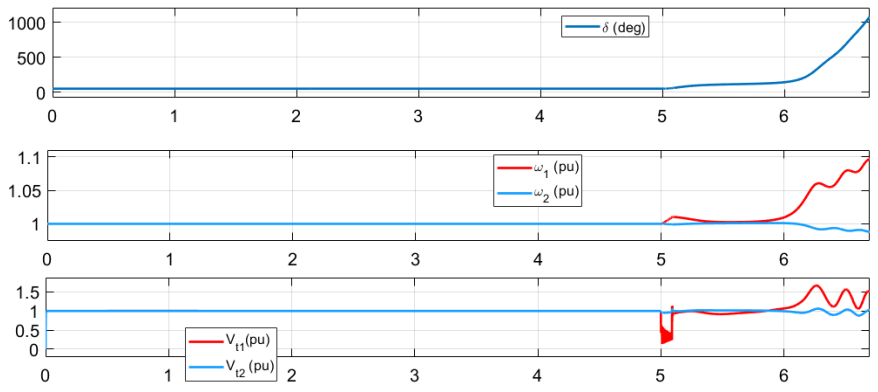

Fig. 3. Standard PSS-AVR: (a) power angle $\delta$ in degrees; (b) angular speeds $\omega_{1,2}$ in p.u.; (c) output voltages $V_{t 1,2}$.

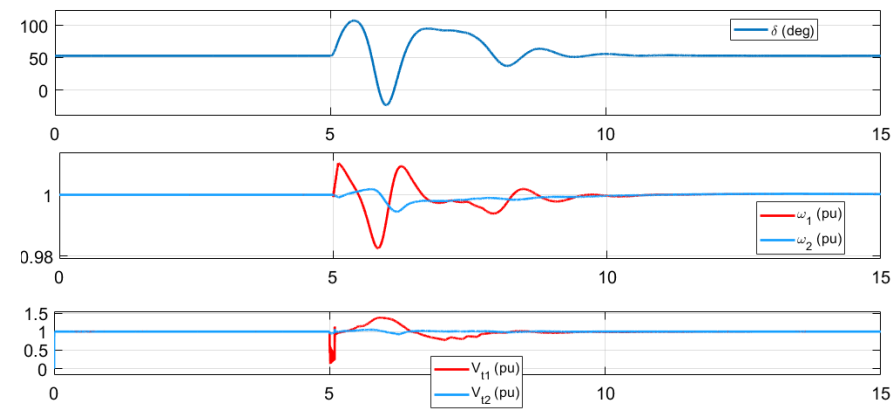

Fig. 4. Output feedback control (15) under noise effects: (a) power angle $\delta$ in degrees; (b) angular speeds $\omega_{1,2}$ in p.u.; (c) output voltages $V_{t 1,2}$ in p.u.

\section{Conclusions}

The transient stabilization and voltage regulation problem for a synchronous generator connected to an infinite bus (see Definition 1) has been addressed when all the physical parameters are uncertain. New interpretations for the DFLR (3)

\footnotetext{
${ }^{5}$ Usually (in similar scenarios) all current structures work pretty well under noise.
} 


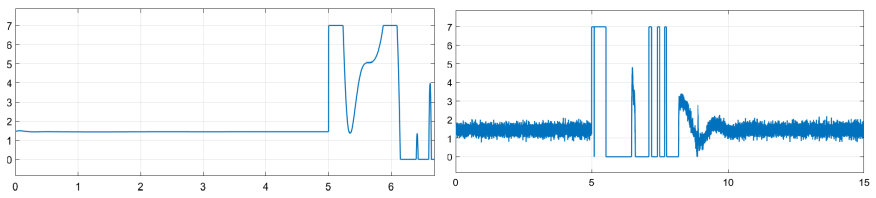

Fig. 5. Standard PSS-AVR (left); output feedback control (15) under noise effects (right): control input in p.u.

have been first derived in Section III (see Lemma 1, Theorem 1 and Corollaries 1-2). Such new interpretations have led to the nonlinear control (15), whose linear action coincides with the one provided by (3): simplicity of control structure (just one integrator is involved) and robust tuning procedure of the linear design (see Theorem 2) have been preserved, with, additionally, no use of the mechanical input power. Control (15) has endowed the robust coordinated PSS-AVR (3) with: i) a nonlinear term that relies on the power angle, allowing for an automatic gain scheduling; ii) nonlinear robustifying terms on the back-stepping-based electrical power tracking error, with the aim of enlarging the machine stability region (as illustrated by numerical analysis). Realistic simulations concerning a benchmark scenario have finally shown that the nonlinear controller preserves the stability when a $90 \mathrm{~ms}-$ long short circuit occurs, whereas instability arises when the standard PSS-AVR is used.

\section{REFERENCES}

[1] P.M. Anderson, A.A. Fouad, Power System Control and Stability, WileyIEEE Press: New York, 2003.

[2] N. Barabanov, J. Schiffer, R. Ortega, D. Efimov, Conditions for almost global attractivity of a synchronous generator connected to an infinite bus, IEEE Transactions on Automatic Control, 62: 4905-4916, 2017.

[3] H. Bourlés, S. Peres, T. Margotin, M.P. Houri, Analysis and design of a robust coordinated AVR/PSS, IEEE Transactions on Power Systems, 13: 568-575, 1998.

[4] G. Damm, F. Lamnabhi-Lagarrigue, R. Marino, C.M. Verrelli, Transient stabilization and voltage regulation of a synchronous generator, in Taming Heterogeneity and Complexity of Embedded Control, Editors: F. Lamnabhi-Lagarrigue, S. Laghrouche, A. Loria and E. Panteley, International Scientific \& Technical Encyclopedia (ISTE), London, 211225, 2007.

[5] G. Damm, R. Marino, F. Lamnabhi-Lagarrigue, Adaptive nonlinear output feedback for transient stabilization and voltage regulation of power generators with unknown parameters, International Journal of Robust and Nonlinear Control, 14: 833-855, 2004.

[6] G.J.W. Dudgeon, W.E. Leithead, A. Dyśko, J. O'Reilly, J.R. McDonald, The effective role of AVR and PSS in power systems: frequency response analysis, IEEE Transactions on Power Systems, 22: 1986-1994, 2007.

[7] A. Dyśko, W.E. Leithead, J. O'Reilly, Enhanced power system stability by coordinated PSS design, IEEE Transactions on Power Systems, 25: 413-422, 2010.

[8] L. Gao, L. Chen, Y. Fan, H. Ma, A nonlinear control design for power systems, Automatica, 28: 975-979, 1992.

[9] J. C. Gonzalez-Torres, G. Damm, V. Costan, A. Benchaib, F. LamnabhiLagarrigue, A novel distributed supplementary control of Multi-Terminal VSC-HVDC grids for rotor angle stability enhancement of AC/DC systems', IEEE Transactions on Power Systems, doi: 10.1109/TPWRS.2020.3030538, October, 2020.

[10] Y. Guo, D.J. Hill, Y. Wang, Global transient stability and voltage regulation for power systems, IEEE Transactions on Power Systems, 16: 678-688, 2001.

[11] J.B. Hoagg, D.S. Bernstein, Direct adaptive dynamic compensation for minimum phase systems with unknown relative degree, IEEE Transactions on Automatic Control, 52: 610-621, 2007.

[12] X. Jiao, Y. Sun, T. Shen, Adaptive controller design for a synchronous generator with unknown perturbation in mechanical power, International Journal of Control, Automation, and Systems, 3: 308-314, 2005.
[13] K.T. Law, D.J. Hill, N.R. Godfrey, Robust co-ordinated AVR-PSS design, IEEE Transactions on Power Systems, 9: 1218-1225, 1994.

[14] J. Machowski, S. Robak, J.W. Bialek, J.R. Bumby, N. Abi-Samra, Decentralized stability-enhancing control of synchronous generator, IEEE Transactions on Power Systems, 15: 1336-1344, 2000.

[15] I. Mareels, A simple selftuning controller for stably invertible systems, Systems \& Control Letters, 4: 5-16, 1984.

[16] R. Marino, T. Shen, C.M. Verrelli, Robust adaptive transient stabilization of a synchronous generator with parameter uncertainty, European Journal of Control, 12: 135-155, 2006.

[17] R. Marino, P. Tomei, C.M. Verrelli, Learning control for nonlinear systems in output feedback form, Systems and Control Letters, 61: 12421247, 2012.

[18] R. Marino, C.M. Verrelli, A global state feedback output regulating control for uncertain systems in strict feedback form, Systems and Control Letters, 58: 682-690, 2009.

[19] H. Quinot, H. Bourlés, T. Margotin, Robust coordinated AVR+PSS for damping large scale power systems, IEEE Transactions on Power Systems, 14: 1446-1451, 1999.

[20] P.W. Sauer, M.A. Pai, Power System Dynamics and Stability, Prentice Hall: Upper Saddle River, 1998.

[21] C.M. Verrelli, G. Damm, Robust transient stabilization problem for a synchronous generator in a power network, International Journal of Control, 83: 816-828, 2010.

[22] C.M. Verrelli, G. Damm, Output feedback transient stabilization and voltage regulation of a synchronous generator, International Journal of Robust and Nonlinear Control, 22: 1495-1504, 2012.

[23] Y. Wang, G. Guo, D.J. Hill, Robust decentralized nonlinear controller design for multimachine power systems, Automatica, 33: 1725-1733, 1997.

[24] E. Weitenberg, C. De Persis, Robustness to noise of distributed averaging integral controllers in power networks, Systems and Control Letters, 119: $1-7,2018$.

[25] E. Weitenberg, C. De Persis, N. Monshizadeh, Exponential convergence under distributed averaging integral frequency control, Automatica, 98: 103-113, 2018.

[26] E. Weitenberg, Y. Jiang, C. Zhao, E. Mallada, F. Dörfler, C. De Persis, Robust decentralized frequency control: a leaky integrator approach, European Control Conference, 764-769, June 12-15, 2018, Limassol, Cyprus.

[27] E. Weitenberg, Y. Jiang, C. Zhao, E. Mallada, C. De Persis, F. Dörfler, Robust decentralized secondary frequency control in power systems: merits and trade offs, IEEE Transactions on Automatic Control, 64(10): 3967-3982, 2019.

[28] C. Zhu, R. Zhou, and Y. Wang, A new nonlinear voltage controller for power systems, Electrical Power and Energy Systems, 19: 19-27, 1997. 